\title{
Clinical efficacy and safety of polymyxins based versus non-polymyxins based therapies in the infections caused by carbapenem-resistant Acinetobacter baumannii: a systematic review and meta- analysis
}

Cheng Lyu ${ }^{1,2+}$, Yuyi Zhang ${ }^{3+}$, Xiaofen Liu ${ }^{1,2}$, Jufang $W^{1}$ and Jing Zhang ${ }^{1,2^{*}}$

\begin{abstract}
Background: The prevalence of infections due to carbapenem-resistant Acinetobacter baumannii (CRAB) is on the rise worldwide. Polymyxins are considered as last-resort drugs for CRAB infections, but there is still controversy regarding the efficacy and safety of polymyxins based therapies in CRAB infections. The present systematic review was designed to compare the efficacy and safety of polymyxins based therapies versus non-polymyxins based therapies in CRAB infections.

Methods: We performed a systematic literature search in PubMed, Embase, CINAHL, Cochrane Library, and clinicaltrials. gov to identify eligible studies reporting the clinical outcomes of patients with CRAB infections. The meta-analysis employed a random-effects model to estimate the odds ratio (OR) and standardized mean difference (SMD) with 95\% confidence interval $(\mathrm{Cl})$. The primary outcome was 1-month mortality for any cause. We also examined clinical response, microbiological response, length of stay in hospital, and adverse events.

Results: Eleven eligible studies were analyzed (1052 patients in total), including 2 randomized clinical trials. Serious risk of bias was found in 8 out of the 11 studies. There was no statistically significant difference between polymyxins based therapies and non-polymyxins based therapies in 1-month mortality for any cause $(\mathrm{OR}, 0.95 ; 95 \% \mathrm{Cl}, 0.59$ to 1.53$)$, microbiological response $(\mathrm{OR}, 3.83 ; 95 \% \mathrm{Cl}, 0.90$ to 16.29$)$ and length of stay in hospital (SMD, $0.24 ; 95 \% \mathrm{Cl},-0.08$ to 0.56$)$. The pooled OR of clinical response indicated a significant difference in favor of polymyxin based therapies (OR, 1.99; 95\% $\mathrm{Cl}, 1.31$ to 3.03). The pooled OR of adverse events showed that non-polymyxins based therapies were associated with fewer adverse events (OR, 4.32; 95\% Cl, 1.39 to 13.48).

(Continued on next page)
\end{abstract}

\footnotetext{
* Correspondence: zhangj_fudan@aliyun.com

${ }^{\dagger}$ Cheng Lyu and Yuyi Zhang contributed equally to this work.

${ }^{1}$ Institute of Antibiotics, Huashan Hospital, Fudan University, 12 Middle Wulumuqi Road, Shanghai 200040, China

${ }^{2}$ Key Laboratory of Clinical Pharmacology of Antibiotics, National Health Commission, Shanghai, China

Full list of author information is available at the end of the article
}

(c) The Author(s). 2020 Open Access This article is licensed under a Creative Commons Attribution 4.0 International License, which permits use, sharing, adaptation, distribution and reproduction in any medium or format, as long as you give appropriate credit to the original author(s) and the source, provide a link to the Creative Commons licence, and indicate if changes were made. The images or other third party material in this article are included in the article's Creative Commons licence, unless indicated otherwise in a credit line to the material. If material is not included in the article's Creative Commons licence and your intended use is not permitted by statutory regulation or exceeds the permitted use, you will need to obtain permission directly from the copyright holder. To view a copy of this licence, visit http://creativecommons.org/licenses/by/4.0/ The Creative Commons Public Domain Dedication waiver (http://creativecommons.org/publicdomain/zero/1.0/) applies to the data made available in this article, unless otherwise stated in a credit line to the data. 
(Continued from previous page)

Conclusion: The performance of polymyxins based therapies was better than non-polymyxin based therapies in clinical response rate and similar to non-polymyxin based therapies in terms of 1-month mortality and microbiological response in treating CRAB infections. Due to the limitations of our study, we cannot draw a firm conclusion on the optimal treatment of CRAB infections, but polymyxins would be a relatively effective treatment for CRAB infections. Adequate and well-designed large scale randomized controlled trials are required to clarify the relative efficacy of polymyxins based and non-polymyxins based therapies.

Keywords: Polymyxin, Colistin, Carbapenem-resistant Acinetobacter baumannii, Meta-analysis

\section{Background}

Acinetobacter baumannii is a gram-negative opportunistic pathogen [1] and a member of the ESKAPE (Enterococcus faecium, Staphylococcus aureus, Klebsiella pneumoniae, Acinetobacter baumannii, Pseudomonas aeruginosa, and Enterobacter) pathogens. A. baumannii can develop diverse mechanisms of resistance and so is capable of escaping from the effects of the commonly used antibiotics [2]. It may cause a range of nosocomial infections, including pneumonia, bacteremia, wound infection, and postneurosurgical meningitis, threatening the lives of patients, particularly in the setting of intensive care unit (ICU) [3].

Carbapenems are considered as the first-line agents for treating A. baumannii infections if the isolates are susceptible. But the widespread use of carbapenems since 1990 has provoked the emergence of carbapenemresistant $A$. baumannii (CRAB) [4]. Lob et al. reported that only about $8-26 \% A$. baumannii isolates were susceptible to carbapenems worldwide [5]. Alarmingly, the prevalence of CRAB isolates increased from $13.3 \%$ in 2004 to $70.5 \%$ in 2014 in China [6].

Polymyxins include polymyxin $\mathrm{B}$ and polymyxin $\mathrm{E}$ (also known as colistin). They were discovered in 1947 [7] but discontinued shortly thereafter due to high nephrotoxicity [8]. In recent years, they are reintroduced into clinical practice for the activity against many multidrug-resistant gram-negative bacilli. Polymyxins may provide a synergistic effect with other antibiotic classes by disrupting the outer membrane of gramnegative bacteria [9].

Currently, polymyxins are the most commonly used agents and often considered as the "last resort" or salvage treatments of CRAB infections [10]. However, there are still many controversies and confusions regarding the efficacy and safety of polymyxins based therapies in treating $\mathrm{CRAB}$ infections. While numerous reports showed good therapeutic effects of polymyxins based therapies [11, 12], some reports linked polymyxins based therapies with a higher mortality $[13,14]$. Other antibiotics, such as tigecycline and sulbactam, alternative therapeutic options against CRAB, also have shown mixed clinical outcomes [15]. Therefore, it is important to elucidate whether the polymyxins based therapies are more effective than other alternative treatments in patients with CRAB infection.

This meta-analysis was designed to evaluate the efficacy and safety of polymyxins based therapies versus non-polymyxins based therapies in CRAB infections based on all the evidence available in the literature.

\section{Methods}

The study design of this systematic review and metaanalysis was consistent with the Preferred Reporting Items for Systematic Reviews and Meta-Analysis Protocols (PRISMA-P 2015) Guidelines [16].

\section{Search strategies}

Databases including PubMed, Embase, CINAHL, Cochrane Library and clinicaltrials.gov (www.clinicaltrials.gov) were searched for all the studies reporting the treatment of infections caused by CRAB from their inception to October 2019. We used the following search string: "polymyxin or colistin" and "Acinetobacter baumannii and drug-resistant" or "Acinetobacter baumannii and carbapenem" or "carbapenem-resistant Acinetobacter baumannii" (see Additional file 1 for detailed search strategy). Furthermore, references listed in the identified articles and other reviews were also searched to select relevant studies. No restrictions of language, publication year or publication status were applied.

\section{Selection criteria}

Studies for inclusion were based on the following criteria: (1) adult patients with CRAB infection, (2) polymyxins group was polymyxins based therapy and control group was non-polymyxins based therapy, (3) randomized controlled trials (RCTs) or cohort studies or casecontrol studies (whether retrospective or prospective), (4) at least one active antibiotic was used in treatment groups, and CRAB isolates were sensitive to polymyxins in the polymyxins group, (5) carbapenem resistance was clearly defined as resistant to imipenem and/or meropenem (without limitation on definition of cut off value), (6) 1-month mortality for any cause was reported. Studies for exclusion were based on the following criteria: (1) animals, in vitro, pharmacokinetics/pharmacodynamics 
(PK/PD) studies or single-arm studies, (2) polymyxins were used in all treatment groups, (3) studies in patients infected with mixed microorganisms, (4) abstracts presented at conferences, editorials, reviews, systematic reviews, and meta-analyses, (5) less than five cases per treatment group were reported. We attempted to contact the authors for details if the data were unclear or missing.

Full-text articles were retrieved for the studies that fulfilled selection criteria. Two authors (CL \& YZ) further checked the eligibility of each study independently.

\section{Outcomes}

In this study, the primary outcome was 1-month (28-30 days) mortality for any cause (1-month mortality). The secondary outcomes were clinical response, microbiological response, length of stay in hospital and adverse events. Clinical response was defined as complete resolution of at least two signs of infection (such as abnormal temperature, leukocytosis or leukopenia) at the end of treatment. The signs of infection varied due to the site of infection. Clinical judgment was made by the clinician according to local guidelines. The microbiological response was defined as a negative microbiological culture, which was obtained at the end of treatment. Length of stay in hospital was measured from the date of the infection was diagnosed to the date of discharge or death. Adverse events included nephrotoxicity, hepatotoxicity, skin rash, and diarrhea.

\section{Data extraction and quality assessment}

The information of first authors, publication years, study years, countries, study designs, patient demographics (including age, gender, resistance profile of the bacteria, disease, and APACHE II score), clinical settings, sample sizes, interventions (including regimen and route of administration) and clinical outcomes were extracted from individual studies. All data extraction was done independently and checked by two authors (CL and YZ) using a pre-defined data extraction form and then compared for verification. The risk of bias of individual studies was assessed by four authors (CL, YZ, XL \& JW) using the Risk of Bias in Non-randomized Studies of Interventions (ROBINS-I) tool endorsed by Cochrane Scientific Committee for observational studies [17]. We assessed the risk of bias for seven domains including bias due to confounding, selection of participants into the study, classification of the intervention, deviations from intended interventions, bias due to missing data, measurement of outcomes, and selection of the reported result. The rating of each domain ranged from low, moderate, serious, to critical risk, and no information (NI). The rating of risk of bias was based on the data we used for meta-analysis rather than the data in publications. Any discrepancies were settled by consensus.

\section{Statistical analysis}

We performed a meta-analysis using the MantelHaenszel method (random-effects model) and inverse variance approach (random-effects model) to estimate the Odd Ratio (OR) and standardized mean difference (SMD) with 95\% confidence interval (CI) to compare polymyxins based therapies with non-polymyxins based therapies. Dichotomous variables including 1-month mortality, clinical response, microbiological response, and adverse events were described by OR and CI. Continuous variable, length of stay in hospital, was described by SMD and CI. Heterogeneity between studies was assessed by the Q statistics and $I^{2}$ tests $\left(P<0.05\right.$ and $I^{2}>$ $50 \%$ suggesting significant heterogeneity). Sensitivity analyses were performed on efficacy outcomes to identify the source of heterogeneity by excluding the studies with serious risk of bias, excluding studies published before 2010, excluding studies with small sample size and excluding studies with inadequate balance in baseline characteristics. The leave-one-out analysis was conducted to ensure that no single study unduly influenced the overall effect size. A funnel plot was used to measure the publication bias. Egger's test and Peters' test were used to evaluate the asymmetry of funnel plot [18]. Subgroup analyses were done to compare the efficacy split by infection site, route of administration and region. All the above analyses were performed with Review Manager (RevMan) software, Version 5.3, (Copenhagen: The Nordic Cochrane Centre, The Cochrane Collaboration, 2014.) except the analysis of publication bias, which was done with STATA software, Version 13.0. (StataCorp LLC, 2013).

\section{Results}

The selection of included studies

A total of 271 studies were identified through the electronic database search and 15 studies were identified through manual search. After removing the duplicate records between databases, the abstracts of the remaining 262 articles were retrieved for a preliminary screening. Thirty-four studies with full texts were further assessed for eligibility, of which 13 studies were excluded because of insufficient information of carbapenem-resistance, 5 were excluded due to the unavailability of the data of 1month mortality, 4 were excluded because patients were co-infected with other bacteria and 1 study was excluded because patients in control group did not receive active treatments. Finally, a total of 11 studies were included in our meta-analysis. Additional data were obtained from the author of the paper published by Raz-Pasteur and colleagues in 2019 [19]. The data were adequate for 
comparing colistin with ampicillin-sulbactam and comparing colistin with trimethoprim-sulfamethoxazole separately in two independent sets of data. Therefore, a total of 12 datasets were analyzed. A flowchart of study selection is shown in Fig. 1.

\section{Study characteristics}

The meta-analysis was performed with 11 studies [13, 14, 19-27], including a total of 1052 patients (496 patients in polymyxins group and 556 patients in control group). The characteristics of the included studies are listed in Table 1. These 11 studies consisted of 8 retrospective studies [13, 14, 19, 22, 24-27], 2 RCTs [20, 21] and 1 prospective study [23]. The research hospitals are in North America and Europe (USA, Greece, Spain, Turkey, and France), Middle East (Iran, and Israel) and Asia (Taiwan, China, and Thailand). Two studies [25, 26] assessed patients with intracranial infections (such as meningitis). One study [13] reported on patients with intra-abdominal infection. Five studies examined pneumonia [14, 20-22, 24], including hospital-acquired pneumonia (HAP) and ventilator-associated pneumonia (VAP). Three studies [19, 23, 27] included more than one site of infection. In polymyxins group, colistin was administered in 10 studies [13, 14, 19-24, 26, 27] and polymyxin B [25] was used in only one study. In the control group, carbapenems, tetracyclines, cephalosporins, $\beta$-lactam $/ \beta$-lactamase inhibitors, quinolones, aminoglycosides and glycylcycline (tigecycline) were the most commonly used alternative antibiotics. In 7 studies $[13,14,19-21,23,24]$, the intravenous (IV) route of administration was used in both polymyxins and control groups. But in the other 4 studies [22, 25-27], patients in polymyxins group received IV and intrathecal/intracerebral (IT) polymyxins or IV and inhaled (IH) polymyxins, while patients in control group were given IV antibiotics only. One-month mortality was reported in all studies, clinical and microbiological response was reported in 5 studies. The length of stay in hospital and adverse events were reported in 4 studies.

\section{Quality assessment}

The output of the ROBINS-I tool is summarized in Fig. 2. The risk of bias was rated as moderate for 2 RCTs $[20,21]$ and one [14] retrospective study, serious for the remaining 8 studies. Seven studies [19, 22-27] were at serious risk of bias in the domain of "bias due to confounding". The common confounding factors were comorbidities (especially immunodeficiency), mixed infection sites, antibiotic susceptibility, severity of disease, and empirical treatment. Six studies [13, 23-27] were judged to be at serious risk in the domain of "bias in classification of interventions" because of lacking records of the dose/loading dose, frequency, and timing of intervention. Only one study was at serious risk of bias due to missing 50 records of patients without explanation [25].

\section{Meta-analysis for 1-month mortality}

Eleven studies reporting the 1-month mortality of polymyxins based therapies and non-polymyxins based therapies for CRAB infections were included in this metaanalysis. There was no statistically significant difference between the two groups in 1-month mortality (OR, 0.95; 95\% CI, 0.59 to $1.53 ; P=0.84)$. Statistically significant heterogeneity $\left(P=0.01, I^{2}=54 \%\right)$ was observed (Fig. 3).

\section{Meta-analysis for clinical response}

Five studies [20-22, 26, 27] involving 449 patients compared the clinical response of two types of therapies. No statistically significant heterogeneity was observed among these studies $\left(P=0.64, I^{2}=0.0 \%\right)$. The pooled OR of clinical response suggested that polymyxins based therapies may have an advantage over non-polymyxins based therapies (OR, 1.99; 95\% CI, 1.31 to $3.03 ; P=$ 0.001) (Fig. 4a).

\section{Meta-analysis for microbiological response}

Five studies [20, 21, 25-27] (261 patients) reported the microbiological response. The microbiological response rates favored polymyxins group, but the difference was not statistically significant (OR, 3.83; $95 \% \mathrm{CI}, 0.90$ to $16.29 ; P=0.07$ ) between the two types of therapies. Statistically significant heterogeneity $\left(P=0.002, I^{2}=76 \%\right)$ was observed among the five studies (Fig. 4b).

\section{Meta-analysis for length of stay in hospital}

Four studies [13, 14, 19, 27] reported the length of stay in hospital. The heterogeneity observed among 4 studies was not statistically significant $\left(P=0.24, I^{2}=28 \%\right)$. The length of stay in hospital did not differ significantly (SMD, 0.24; 95\% CI, -0.08 to $0.56 ; P=0.14$ ) between polymyxins based therapies (210 patients) and nonpolymyxins based therapies (81 patients), but with a trend of prolonged hospitalization for patients receiving polymyxins based therapies (Fig. 4c).

\section{Meta-analysis for adverse events}

Adverse events were recorded in 4 studies [13, 20, 21, 26] involving 123 patients. The main adverse events of polymyxins based therapies were associated with nephrotoxicity. Twenty nephrotoxicity-related adverse events were seen in the 61 patients with polymyxins based therapies, and 6 in the 62 patients treated with non-polymyxins based therapies. Our result showed that more adverse events occurred in polymyxins group than in control group (OR, 4.32; 95\% CI, 1.39 to 13.48; $P=0.01$ ) (Fig. 4d). Statistical heterogeneity was not significant among these studies $\left(P=0.29, I^{2}=21 \%\right)$. 


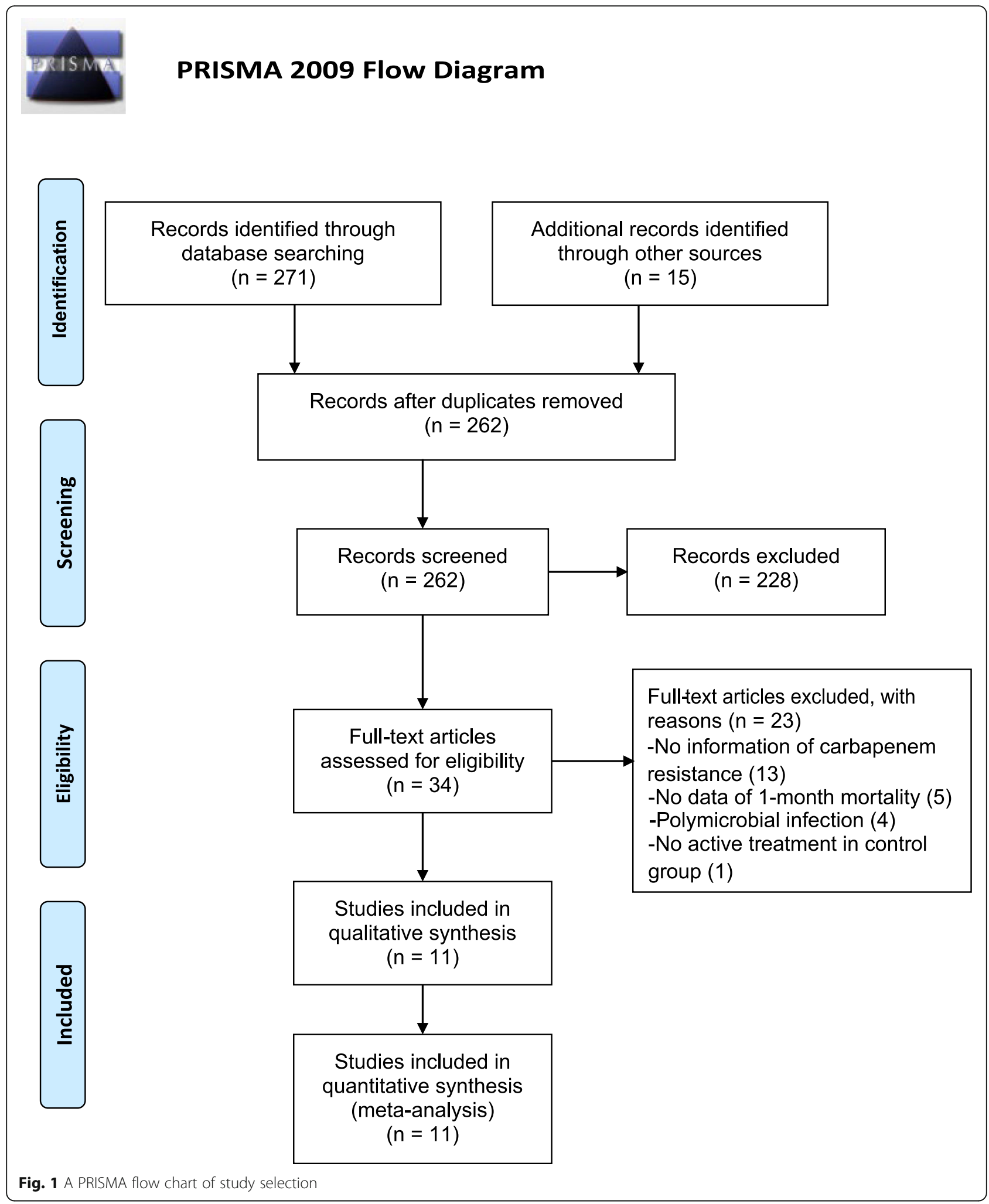

Sensitivity analyses and publication bias analysis

We performed four sensitivity analyses to identify the source of heterogeneity by subgrouping. The planned exclusion was removal of studies with serious risk of bias, removal of studies published before 2010, removal of studies with small sample size, and removal of studies with inadequate balance in the baseline characteristics. The primary outcome and the two secondary outcomes 


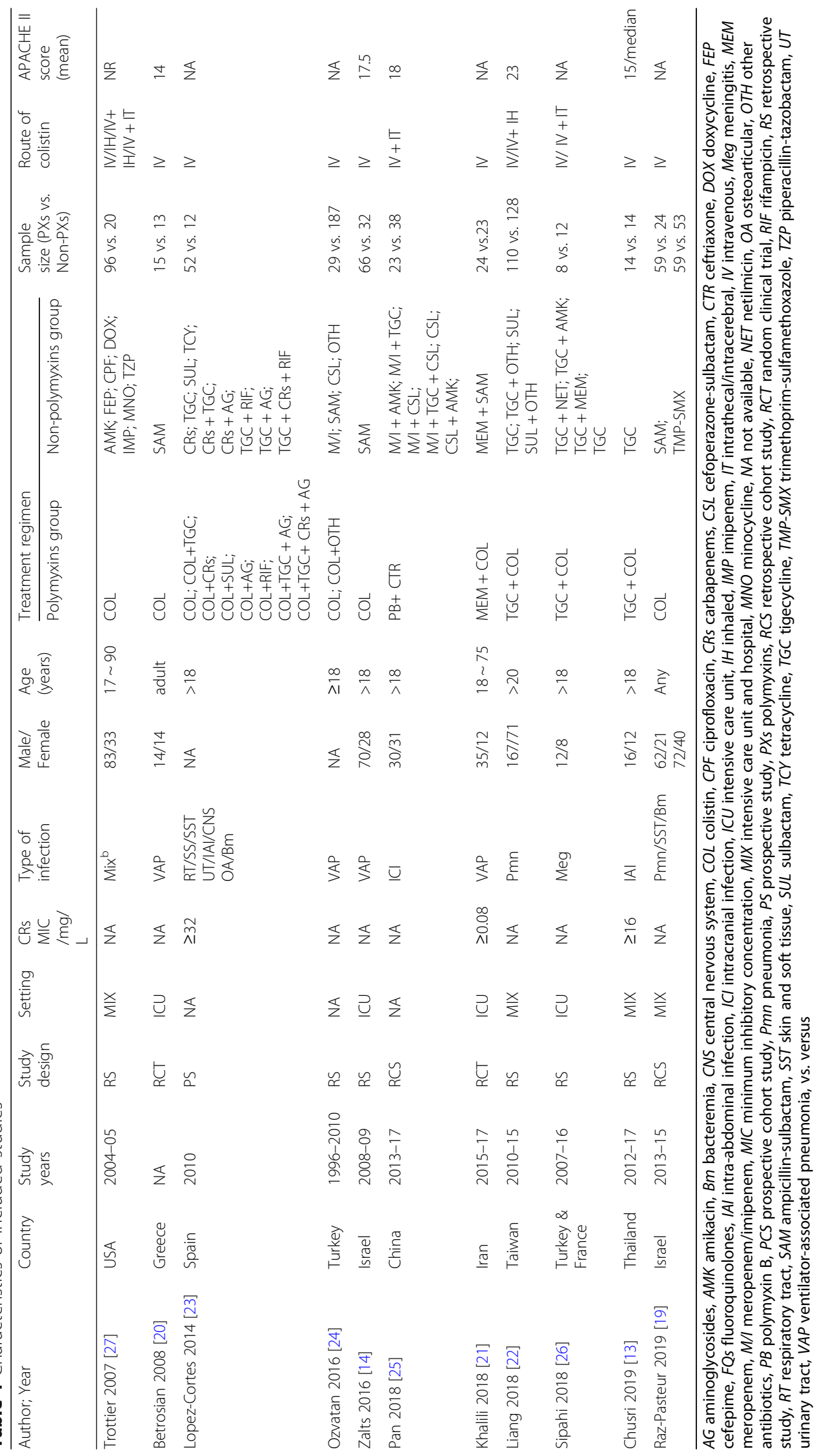




\section{A. Summary of risk of bias of included studies}

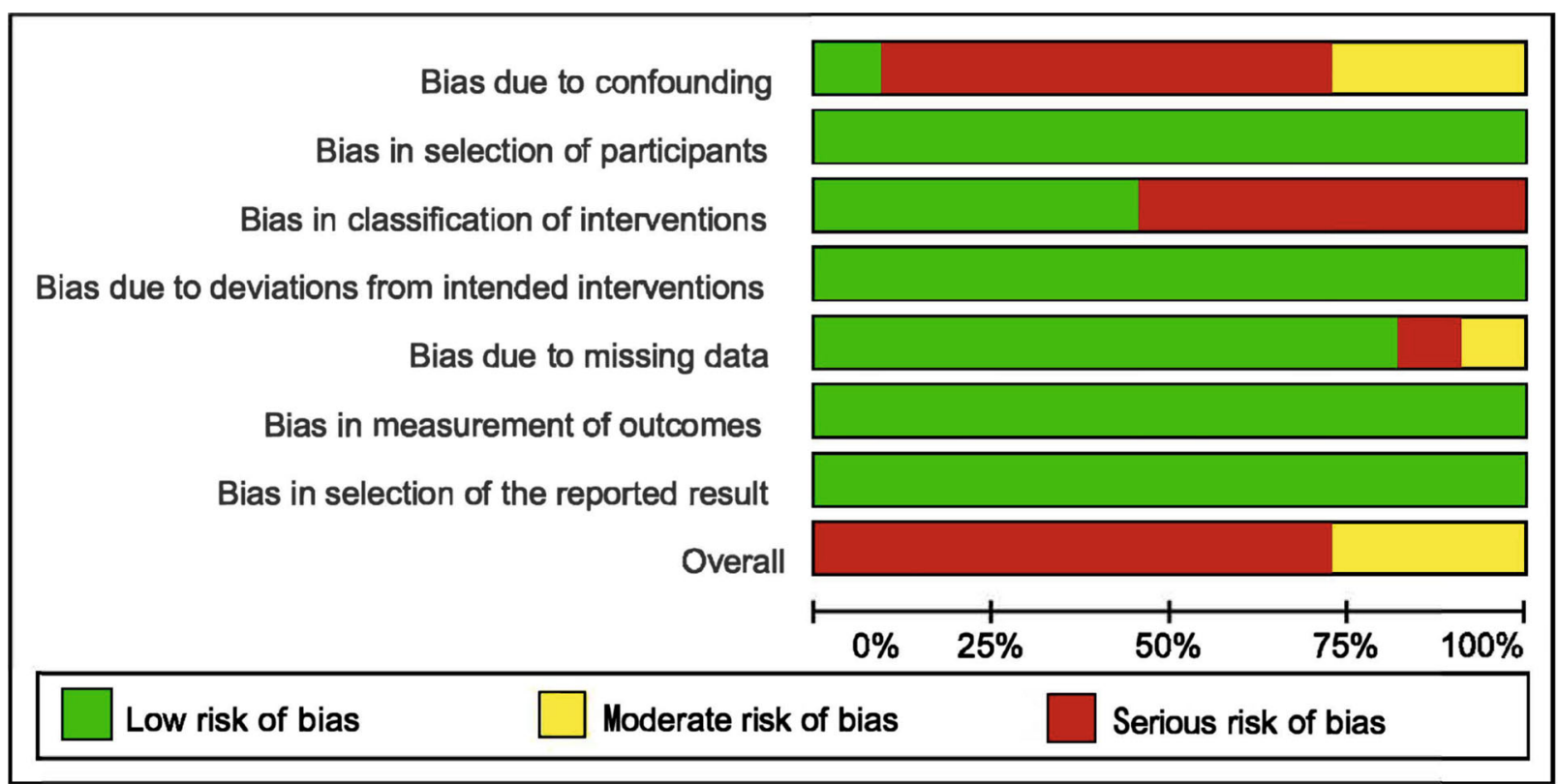

\section{B. Risk of bias of individual studies}

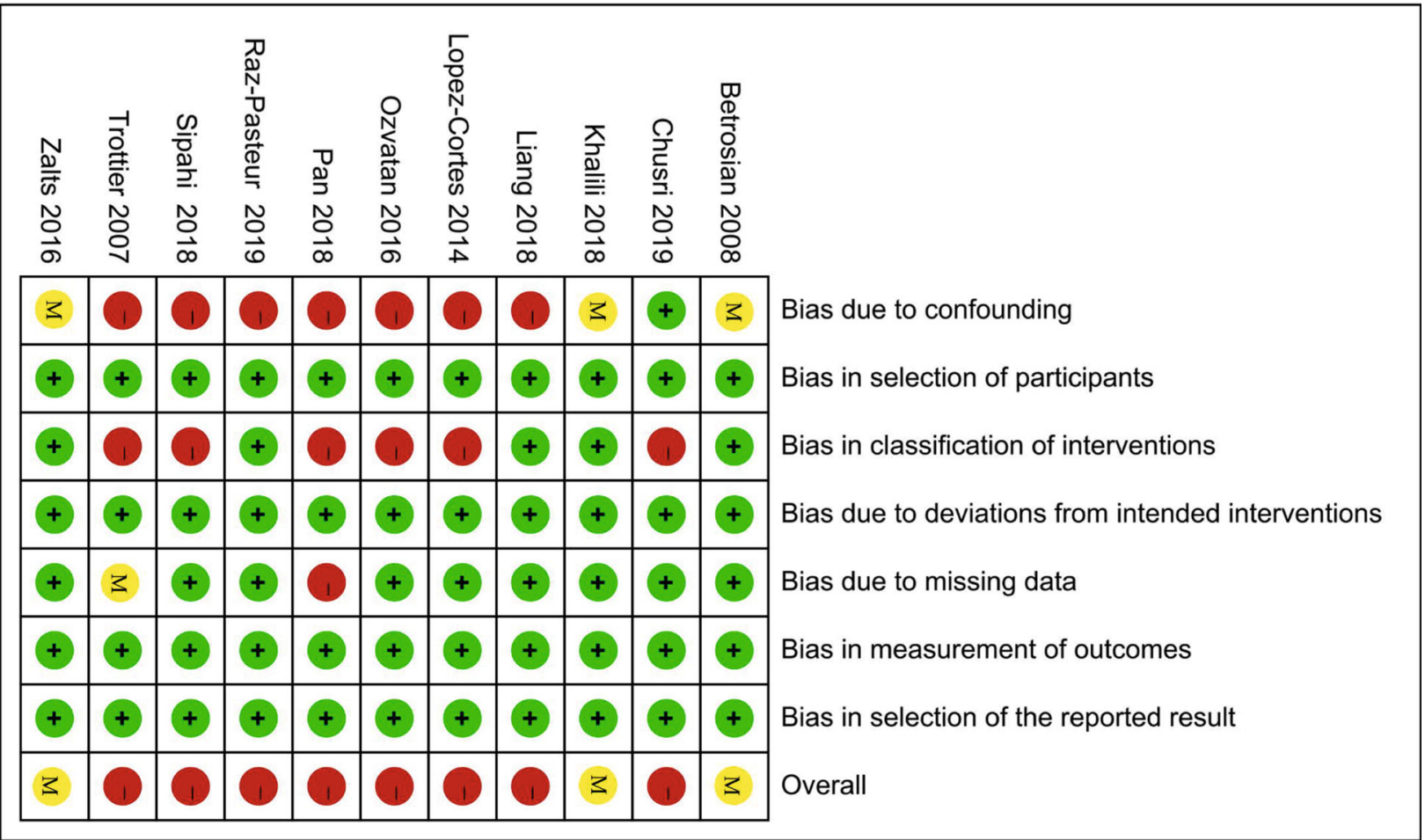

Fig. 2 Summary of risk of bias: a Summary of risk of bias of included studies; $\mathbf{b}$ Risk of bias in individual studies. The green, yellow and red represent "low risk of bias", "moderate risk of bias" and "serious risk of bias", respectively 


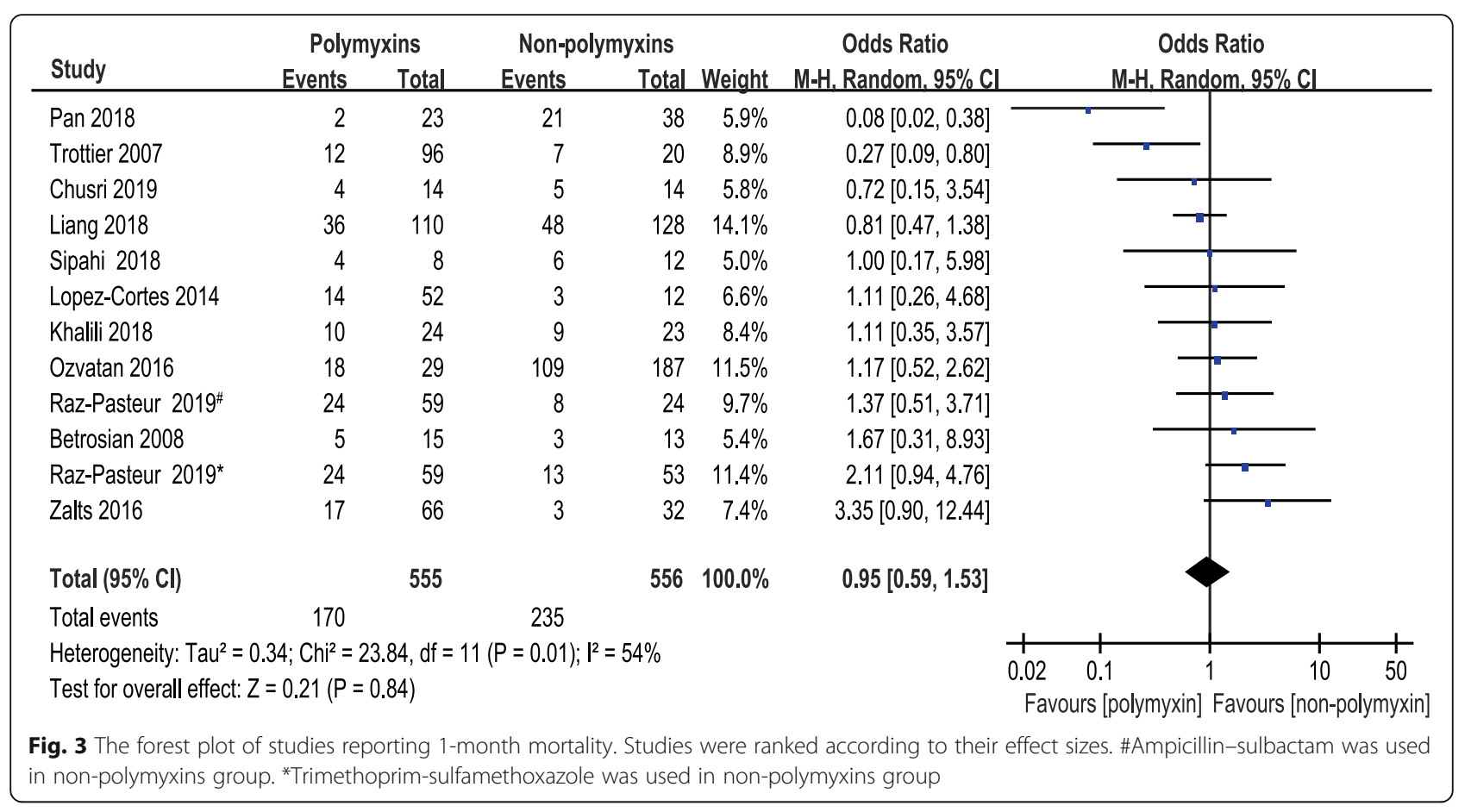

(clinical response and adverse events) did not change substantially after subgrouping, which proved the consistency of our results (Additional file 2: Table S1).

A leave-one-out analysis was also performed to reflect the effect of individual dataset on the pooled ORs. The corresponding pooled ORs of our primary outcome were not changed remarkably (Additional file 2: Table S2).

The funnel plot was constructed to assess the publication bias of the literature (Additional file 3). There was no evidence of publication bias based on Egger's test $(t=-1.21 ; P=0.253)$ and Peters' test $(t=-0.18 ; P=$ $0.864)$. The tests could be underpowered due to small sample size.

\section{Subgroup analyses}

Subgroup analyses of 1-month mortality were planned to split the studies according to infection site, route of administration or region. Studies were classified into 4 subgroups in terms of infection site, i.e., pneumonia, mixed infection, intracranial infection, and intraabdominal infection. The pooled ORs of 1-month mortality did not differ from the primary analysis remarkably. No significant difference was seen between polymyxins based therapies and non-polymyxins based therapies after subgrouping. The pooled ORs of pneumonia, intracranial infection and mixed infection were 1.10 (95\% CI, 0.73 to 1.66 ), 0.27 (95\% CI, 0.02 to 3.35 ) and 0.99 (95\% CI, 0.39 to 2.50), respectively. Only one paper reported the intra-abdominal infection caused by CRAB and the OR was 0.72 with $95 \%$ CI (0.15 to 3.54) (Fig. 5). A subgroup analysis was carried out based on route of administration to compare the 1-month mortality between a subset of studies in which antibiotics were given intravenously in both groups and a subset of studies in which polymyxins were given by multiple routes. The pooled OR of studies, in which multiple routes (IV plus IH/IT) were used for polymyxins administration, was 0.33 (95\% CI, 0.11 to 0.96 ). The pooled OR was 1.25 (95\% CI, 0.85 to 1.83 ) in studies that antibiotics were administered intravenously only (Fig. 6). This result indicated that lower mortality was associated with the direct delivery of polymyxins to the focus of infection by intrathecal/intracerebral ventricle injection or inhalation. A subgroup analysis was also carried out according to geographical region in order to understand the regional difference of 1-month mortality. Interestingly, the result showed that studies from Middle East region were in favor of non-polymyxin therapies because of lower mortality rate (OR, 1.79; 95\% CI, 1.07 to 2.98) (Fig. 7).

\section{Discussion}

Infections due to $C R A B$ require special attention. The high morbidity/mortality, the potential to cause outbreaks and the spread of antibiotic resistance are all associated with CRAB infections [3]. Polymyxins are the common options for CRAB infections in clinical practice nowadays. Are polymyxins actually the optimal choice for treating CRAB infections? We aim to answer this question by compiling the up-to-date knowledge on the efficacy and safety of polymyxin-based and nonpolymyxin-base therapies for CRAB infections. 


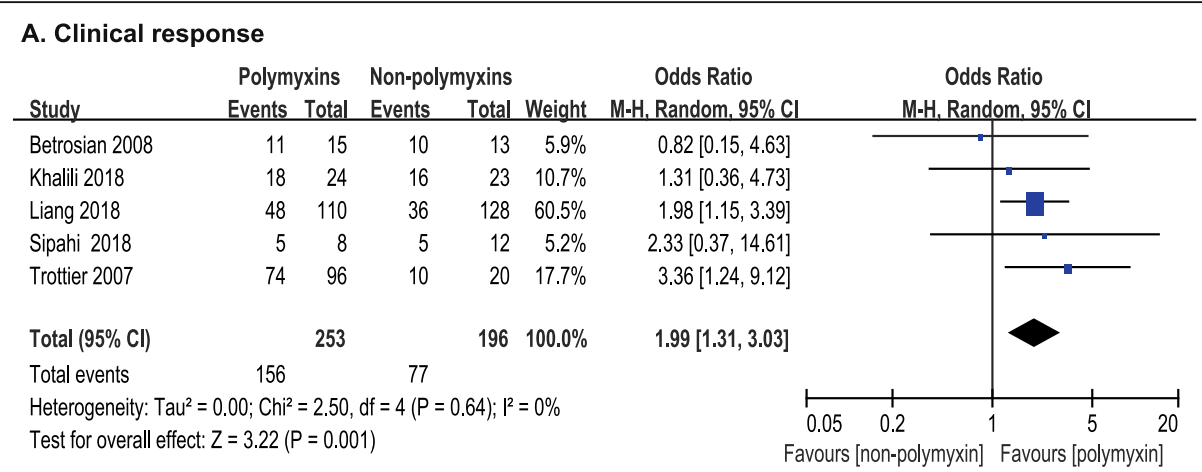

\section{B. Microbiological response}

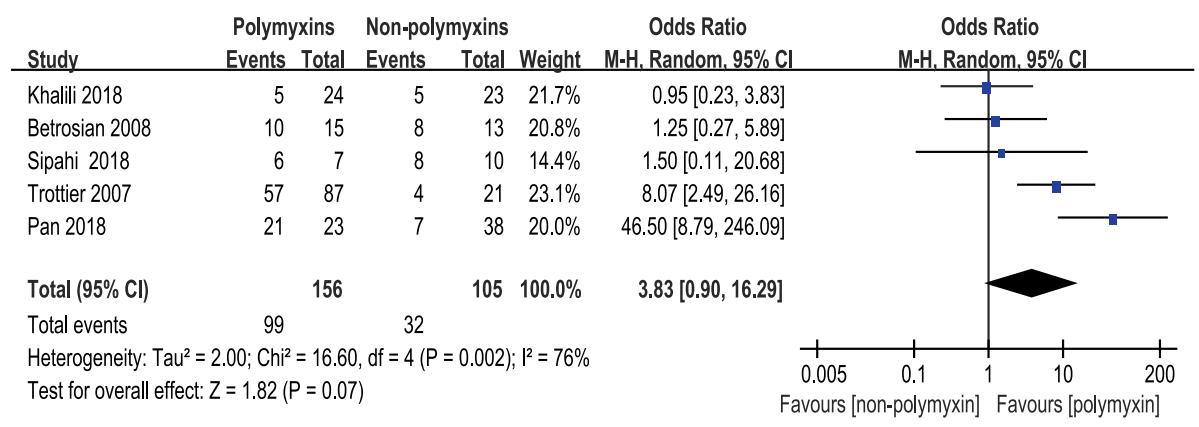

\section{Length of stay in hospital}

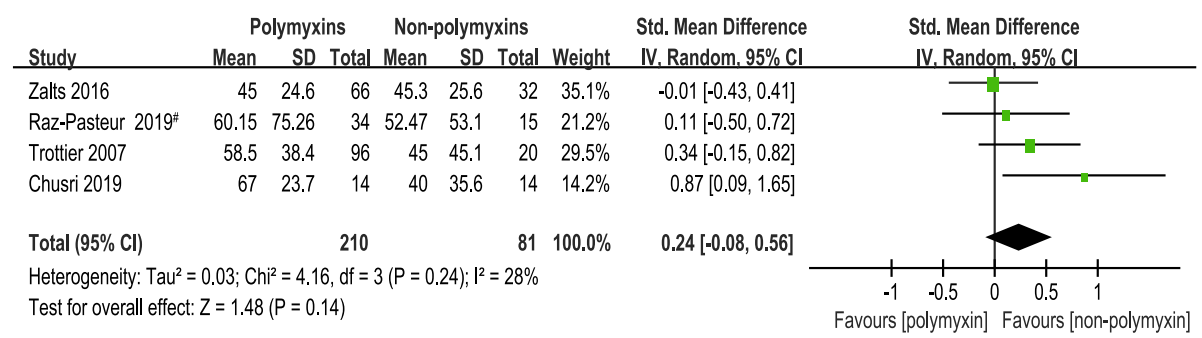

\section{Adverse events}

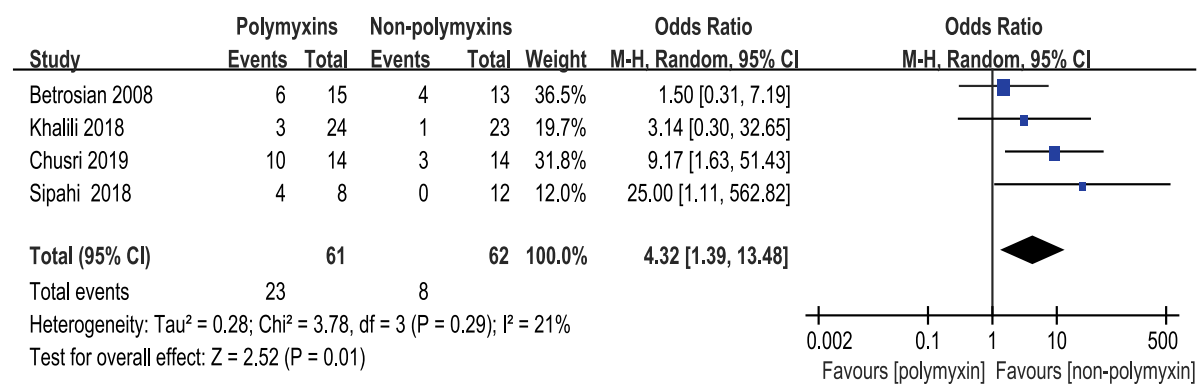

Fig. 4 The forest plots of secondary outcomes: a clinical response; $\mathbf{b}$ microbiological response; c Length of stay in hospital; $\mathbf{d}$ Adverse events. Studies were ranked according to their effect sizes. \#Ampicillin-sulbactam was used in non-polymyxins group

The goal of all medical treatments is to improve the overall survival of patients. The all-cause mortality is a measurement of overall survival and an objective endpoint which is not subject to bias. That is why we chose 1-month mortality for any cause as the primary efficacy outcome in this meta-analysis. And one-month followup period is commonly used in hospitals [28]. One- month mortality for any cause was $30.6 \%$ in polymyxins group and $42.3 \%$ in non-polymyxins group. Our metaanalysis showed that polymyxins based therapies were not associated with lower 1-month mortality when comparing with non-polymyxins based therapies. Many uncertainties are related to 1 -month mortality. Especially in our study, 193 patients were in ICU settings with 


\begin{tabular}{|c|c|c|c|c|c|c|c|}
\hline \multirow[b]{2}{*}{ Study or Subgroup } & \multicolumn{2}{|c|}{ Polymyxins } & \multicolumn{3}{|c|}{ Non-polymyxins } & \multirow{2}{*}{$\begin{array}{c}\text { Odds Ratio } \\
\text { M-H, Random, } 95 \% \mathrm{Cl}\end{array}$} & \multirow{2}{*}{$\begin{array}{c}\text { Odds Ratio } \\
\text { M-H, Random, } 95 \% \mathrm{Cl}\end{array}$} \\
\hline & Events & Total & Events & Total & Weight & & \\
\hline \multicolumn{8}{|l|}{ 1. Pneumonia } \\
\hline Liang 2018 & 36 & 110 & 48 & 128 & $48.5 \%$ & $0.81[0.47,1.38]$ & \\
\hline Khalili 2018 & 10 & 24 & 9 & 23 & $12.0 \%$ & $1.11[0.35,3.57]$ & \\
\hline Ozvatan 2016 & 18 & 29 & 109 & 187 & $24.0 \%$ & $1.17[0.52,2.62]$ & \\
\hline Betrosian 2008 & 5 & 15 & 3 & 13 & $5.9 \%$ & $1.67[0.31,8.93]$ & \\
\hline Zalts 2016 & 17 & 66 & 3 & 32 & $9.6 \%$ & $3.35[0.90,12.44]$ & \\
\hline Subtotal $(95 \% \mathrm{Cl})$ & & 244 & & 383 & $100.0 \%$ & $1.10[0.73,1.66]$ & \\
\hline Total events & 86 & & 172 & & & & \\
\hline \multicolumn{8}{|c|}{ Heterogeneity: $\operatorname{Tau}^{2}=0.02 ; \mathrm{Chi}^{2}=4.29, \mathrm{df}=4(\mathrm{P}=0.37) ; \mathrm{I}^{2}=7 \%$} \\
\hline \multicolumn{8}{|c|}{ Test for overall effect: $Z=0.45(P=0.65)$} \\
\hline \multicolumn{8}{|c|}{ 2. Intracranial infection } \\
\hline Pan 2018 & 2 & 23 & 21 & 38 & $51.4 \%$ & $0.08[0.02,0.38]$ & \\
\hline Sipahi 2018 & 4 & 8 & 6 & 12 & $48.6 \%$ & $1.00[0.17,5.98]$ & \\
\hline Subtotal $(95 \% \mathrm{Cl})$ & & 31 & & 50 & $100.0 \%$ & $0.27[0.02,3.35]$ & \\
\hline Total events & 6 & & 27 & & & & \\
\hline \multicolumn{8}{|c|}{ Heterogeneity: $\mathrm{Tau}^{2}=2.58 ; \mathrm{Chi}^{2}=4.46, \mathrm{df}=1(\mathrm{P}=0.03) ; \mathrm{I}^{2}=78 \%$} \\
\hline \multicolumn{8}{|c|}{ Test for overall effect: $Z=1.02(P=0.31)$} \\
\hline \multicolumn{8}{|l|}{ 3. Mixed infections } \\
\hline Trottier 2007 & 10 & 87 & 7 & 21 & $24.4 \%$ & $0.26[0.08,0.80]$ & \\
\hline Lopez-Cortes 2014 & 14 & 52 & 3 & 12 & $19.7 \%$ & $1.11[0.26,4.68]$ & \\
\hline Raz-Pasteur 2019\# & 24 & 59 & 8 & 24 & $26.4 \%$ & $1.37[0.51,3.71]$ & \\
\hline Raz-Pasteur $2019^{*}$ & 24 & 59 & 13 & 53 & $29.4 \%$ & $2.11[0.94,4.76]$ & \\
\hline Subtotal $(95 \% \mathrm{Cl})$ & & 257 & & 110 & $100.0 \%$ & $0.99[0.39,2.50]$ & \\
\hline Total events & 72 & & 31 & & & & \\
\hline \multicolumn{8}{|c|}{ Heterogeneity: $\operatorname{Tau}^{2}=0.58 ; \mathrm{Chi}^{2}=9.03, \mathrm{df}=3(P=0.03) ; 1^{2}=67 \%$} \\
\hline \multicolumn{8}{|c|}{ Test for overall effect: $Z=0.01(P=0.99)$} \\
\hline & & & & & & & $+\frac{1}{10}$ \\
\hline & & & & & & & 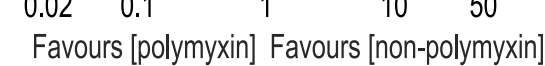 \\
\hline
\end{tabular}

serious comorbidities and complications. Many unknown and undocumented factors could influence the outcome of 1-month mortality.

To further comprehend the relative efficacy on a more specific level, clinical and microbiological responses were designed as secondary outcomes. Our meta-analysis demonstrated that the performance of polymyxins based therapies (desirable clinical response in $61.7 \%$ patients) was better than non-polymyxins based therapies (desirable clinical response in $39.3 \%$ patients) in terms of clinical response. The percentage of patients achieving a good microbiological response at the end of treatment was $63.5 \%$ in polymyxins group, which was 2 times higher than that in non-polymyxins based group
(30.5\%). The microbiological response rates favored polymyxins group, but the statistical significance was not reached, probably due to small sample size.

Just like previous reports [29], we found that more adverse events were reported in polymyxins group, especially nephrotoxic events. A total of 23 adverse events were recorded in polymyxins group, of which 20 were associated with nephrotoxicity. We cannot emphasize enough the importance of renal function monitoring and timely dose adjustment for patients receiving polymyxins. The key was to follow the guidelines of polymyxins usage to balance the efficacy and safety [30, 31].

The subgroup analysis based on route of administration showed that direct delivery of polymyxins to the 


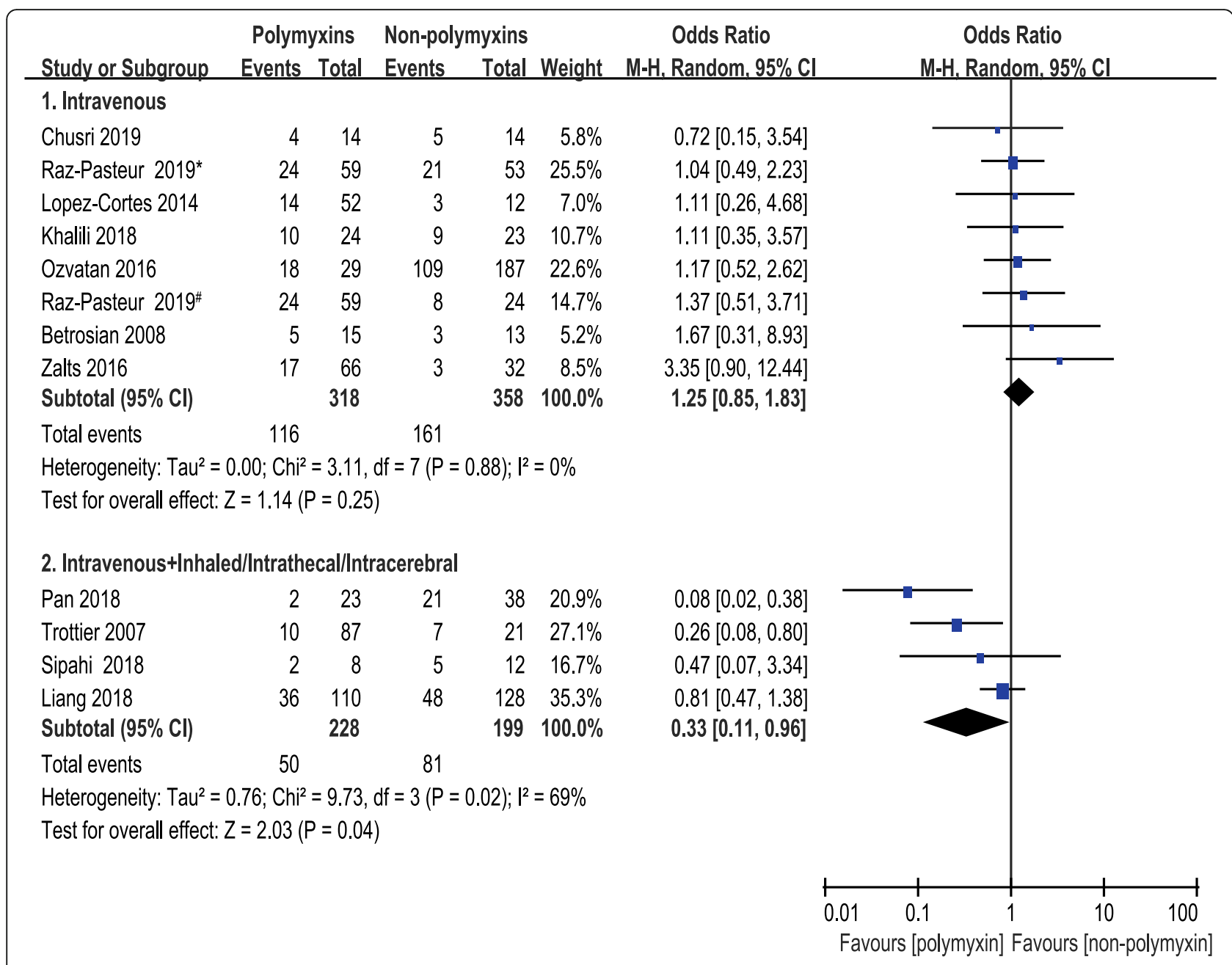

Fig. 6 The forest plot of studies reporting 1-month mortality grouped by route of administration. Studies were ranked according to their effect sizes. \# Ampicillin-sulbactam was used in non-polymyxins group. *Trimethoprim-sulfamethoxazole was used in non-polymyxins group

focus of infection helps. This was in agreement with the previous studies reporting the efficacy of IV polymyxins combined with inhaled or intracranial polymyxins, which was significantly superior to IV polymyxins alone for treating CRAB induced pneumonia and meningitis [3234]. Limited penetration into alveoli and limited ability to cross the blood-brain barrier of polymyxins can partly explain our findings [35, 36].

A previous meta-analysis by Liu et al. in 2014 noted that polymyxins may be effective for treatment of $A$. baumannii infection [37]. However, the cases included in their analysis did not share a clear definition of carbapenem resistance. They evaluated the treatments of $A$. baumannii infections showing different types of drug resistance or no resistance. Given the importance of distinguishing drug resistance in antimicrobial treatments, a clear definition of drug resistance in our study selection could reduce the heterogeneity of meta-analysis. Furthermore, only the studies involving adult patients were included in our analysis, while Liu et al. compiled studies of both adult and pediatric patients. Considering the unique physiological and microbiological conditions of pediatric patients, antimicrobial therapy targeting resistant microorganisms should be discussed in pediatric patients separately. This report is therefore a more robust meta-analysis with improved study design.

Several limitations exist in our meta-analysis. First, the quality of the included studies was low. Only 3 of the 11 studies were at moderate risk of bias and the others were at serious risk of bias. Second, the studied population was heterogeneous. The diverse baseline conditions of infections and comorbidities introduced unknown confounding factors. Third, lacking information about drug use for comorbidity may cause the underestimation of unknown drug-drug interaction which affected the clinical outcomes. Furthermore, the sample size was small which could reduce the power of our analysis. Taking together, the findings must be interpreted with caution. 


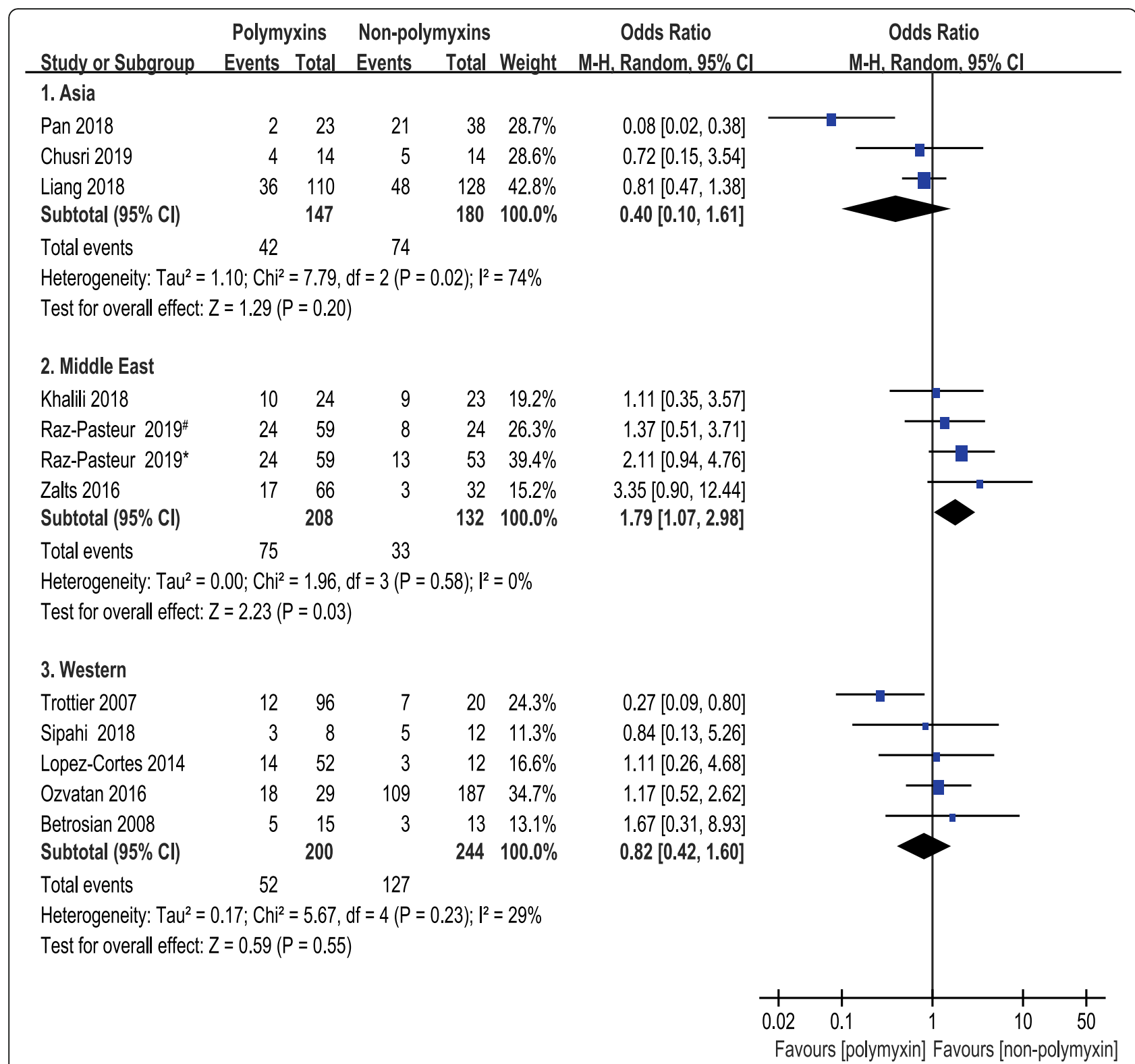

Fig. 7 The forest plot of studies reporting 1-month mortality grouped by geographic region. Studies were ranked according to their effect sizes. \#Ampicillin-sulbactam was used in non-polymyxins group. *Trimethoprim-sulfamethoxazole was used in non-polymyxins group

Our results may be not representative enough for the whole picture of the polymyxins based and nonpolymyxins based therapies against CRAB infections.

\section{Conclusion}

Although our study cannot provide an answer for the optimal treatment of CRAB infection, our results did show that polymyxin could be a relatively effective therapy for CRAB infection. More randomized trials are needed to address the exact efficacy of different antiCRAB treatments. Renal function monitoring to adjust the dose of polymyxin accordingly could largely improve the safety of polymyxin treatment [31]. Stewardship of polymyxin use also helps to prevent the emergence of polymyxin-resistant bacteria. More importantly, all healthcare providers should work closely to keep prioritizing research and development of new antibiotics.

\section{Supplementary information}

Supplementary information accompanies this paper at https://doi.org/10. 1186/s12879-020-05026-2.

Additional file 1. Search Strategies.

Additional file 2: Table S1. Summary of sensitivity analyses. Table S2. Summary of leave-one-out analysis on primary outcome. 
Additional file 3. Funnel plot showing publication bias of 1-month mortality.

\section{Abbreviations}

Cl: Confidence interval; CRAB: Carbapenem-resistant Acinetobacter baumannii; ESKAPE: Enterococcus faecium, Staphylococcus aureus, Klebsiella pneumoniae, Acinetobacter baumannii, Pseudomonas aeruginosa, and Enterobacter spp; HAP: Hospital-acquired pneumonia; ICU: Intensive care unit; IH: Inhaled; IT: Intrathecal/intracerebral; IV: Intravenous; NI: No information; OR: Odds ratio; PK/PD: Pharmacokinetics/pharmacodynamics; RCT: Randomized controlled trial; ROBINS-I: Risk of Bias In Non-randomized Studies of Interventions; SMD: Standardized mean difference; VAP: Ventilator-associated pneumonia

\section{Acknowledgments}

We thank Baoxia Zhang, Ye Cao and Chang Liu for assistance with metaanalysis methodology and text editing that greatly improved the manuscript. We would also like to show our gratitude to the Prof. Paul at The Ruth and Bruce Rappaport Faculty of Medicine, Prof. Trottier at Leonard Miller School of Medicine, Prof. Lopez-Cortes at Hospitales Universitarios Virgen Macarena y Virgen del Rocío, Prof. Akalin at Uludag University, and Prof. Zhang at Zhejiang University School of Medicine for sharing their additional data with us during the course of this study.

\section{Authors' contributions}

This study was designed, coordinated and supervised by JZ as the corresponding author. $\mathrm{CL}$ and $\mathrm{YZ}$ were involved in the study design, data extraction, quality assessment, and data analysis. $\mathrm{XL}$ and JW provided technical assistance for quality assessment. The manuscript was written by $\mathrm{CL}$ and $\mathrm{YZ}$. All authors have read and approved this manuscript. $\mathrm{CL}$ and $\mathrm{YZ}$ contributed equally to this work.

\section{Funding}

This work was supported by the National Major Scientific and Technological Special Project for "Significant New Drugs Development" during the Thirteenth Five-year Plan Period of China (2017ZX09304005). This funder had no role in the study design, data analyses, result interpretation and decision to submit this paper.

\section{Availability of data and materials}

The datasets used and/or analyzed during the current study are available from the corresponding author on reasonable request.

\section{Ethics approval and consent to participate} Not applicable.

\section{Consent for publication}

Not applicable.

\section{Competing interests}

The authors declare that they have no competing interests.

\section{Author details}

'Institute of Antibiotics, Huashan Hospital, Fudan University, 12 Middle Wulumuqi Road, Shanghai 200040, China. ${ }^{2}$ Key Laboratory of Clinical Pharmacology of Antibiotics, National Health Commission, Shanghai, China. ${ }^{3}$ Shanghai Public Health Clinical Center, Shanghai, China.

Received: 20 November 2019 Accepted: 12 April 2020 Published online: 21 April 2020

\section{References}

1. Howard A, O'Donoghue M, Feeney A, Sleator RD. Acinetobacter baumannii: an emerging opportunistic pathogen. Virulence. 2012;3:243-50.

2. Santaiit S, Indrawattana N. Mechanisms of antimicrobial resistance in ESKAPE pathogens. Biomed Res Int. 2016;2016:2475067.

3. Maragakis LL, Perl TM. Acinetobacter baumannii: epidemiology, antimicrobial resistance, and treatment options. Clin Infect Dis. 2008;46:1254-63.
4. Asif M, Alvi IA, Rehman SU. Insight into Acinetobacter baumannii: pathogenesis, global resistance, mechanisms of resistance, treatment options, and alternative modalities. Infect Drug Resist. 2018;11:1249-60.

5. Lob SH, Hoban DJ, Sahm DF, Badal RE. Regional differences and trends in antimicrobial susceptibility of Acinetobacter baumannii. Int J Antimicrob Agents. 2016:47:317-23.

6. Gao L, Lyu Y, Li Y. Trends in drug resistance of Acinetobacter baumannii over a 10-year period: Nationwide data from the China surveillance of antimicrobial resistance program. Chin Med J. 2017;130:659-64.

7. Storm DR, Rosenthal KS, Swanson PE. Polymyxin and related peptide antibiotics. Annu Rev Biochem. 1977:46:723-63.

8. Ordooei Javan A, Shokouhi S, Sahraei Z. A review on colistin nephrotoxicity. Eur J Clin Pharmacol. 2015;71:801-10.

9. Li J, Nation RL, Turnidge JD, Milne RW, Coulthard K, Rayner CR, et al. Colistin: the re-emerging antibiotic for multidrug-resistant gram-negative bacterial infections. Lancet Infect Dis. 2006;6:589-601.

10. Karaiskos I, Lagou S, Pontikis K, Rapti V, Poulakou G. The "old" and the "new" antibiotics for MDR gram-negative pathogens: for whom, when, and how. Front Public Health. 2019;7:151. https://doi.org/10.3389/fpubh.2019.00151.

11. Assimakopoulos SF, Karamouzos V, Lefkaditi A, Sklavou C, Kolonitsiou F, Christofidou M, et al. Triple combination therapy with high-dose ampicillin/ sulbactam, high-dose tigecycline and colistin in the treatment of ventilatorassociated pneumonia caused by pan-drug resistant Acinetobacter baumannii: a case series study. Infez Med. 2019;27:11-6.

12. Cascio A, Conti A, Sinardi L, laria C, Angileri FF, Stassi G, et al. Postneurosurgical multidrug-resistant Acinetobacter baumannii meningitis successfully treated with intrathecal colistin. A new case and a systematic review of the literature. Int J Infect Dis. 2010;14:e572-9. https://doi.org/10. 1016/j.jijid.2009.06.032.

13. Chusri S, Singkhamanan K, Wanitsuwan W, Suphasynth Y, Kositpantawong $\mathrm{N}$, Panthuwong $\mathrm{S}$, et al. Adjunctive therapy of intravenous colistin to intravenous tigecycline for adult patients with non-bacteremic post-surgical intra-abdominal infection due to carbapenem-resistant Acinetobacter baumannii. J Infect Chemother. 2019;25:681-6.

14. Zalts R, Neuberger A, Hussein K, Raz-Pasteur A, Geffen Y, Mashiach T, et al. Treatment of Carbapenem-resistant Acinetobacter baumannii ventilatorassociated pneumonia: retrospective comparison between intravenous Colistin and intravenous ampicillin-Sulbactam. Am J Ther. 2016;23:e78-85. https://doi.org/10.1097/MJT.0b013e3182a32df3.

15. De Pascale G, Montini L, Pennisi M, Bernini V, Maviglia R, Bello G, et al. High dose tigecycline in critically ill patients with severe infections due to multidrug-resistant bacteria. Crit Care. 2014;18:R90. https://doi.org/10.1186/ cc13858.

16. Shamseer $L$, Moher D, Clarke M, Ghersi D, Liberati A, Petticrew M, et al; PRISMA-P Group. Preferred reporting items for systematic review and metaanalysis protocols (PRISMA-P) 2015: elaboration and explanation. BMJ. 2015; 350:g7647. doi: https://doi.org/10.1136/bmj.g7647.

17. Sterne JA, Hernán MA, Reeves BC, Savović J, Berkman ND, Viswanathan M, et al. ROBINS-I: a tool for assessing risk of bias in non-randomised studies of interventions. BMJ. 2016;355:i4919. https://doi.org/10.1136/bmj.i4919.

18. Egger M, Davey Smith G, Schneider M, Minder C. Bias in meta-analysis detected by a simple, graphical test. BMJ. 1997;315:629-34.

19. Raz-Pasteur A, Liron Y, Amir-Ronen R, Abdelgani S, Ohanyan A, Geffen Y, et al. Trimethoprim-sulfamethoxazole vs. colistin or ampicillin-sulbactam for the treatment of carbapenem-resistant Acinetobacter baumannii: a retrospective matched cohort study. J Glob Antimicrob Resist. 2019;17:168-72.

20. Betrosian AP, Frantzeskaki F, Xanthaki A, Douzinas EE. Efficacy and safety of high-dose ampicillin/sulbactam vs. colistin as monotherapy for the treatment of multidrug resistant Acinetobacter baumannii ventilatorassociated pneumonia. J Inf Secur. 2008:56:432-6.

21. Khalili H, Shojaei L, Mohammadi M, Beigmohammadi MT, Abdollahi A Doomanlou M. Meropenem/colistin versus meropenem/ampicillinsulbactam in the treatment of carbapenem-resistant pneumonia. J Comp Eff Res. 2018;7:901-11.

22. Liang CA, Lin YC, Lu PL, Chen HC, Chang HL, Sheu CC. Antibiotic strategies and clinical outcomes in critically ill patients with pneumonia caused by carbapenem-resistant Acinetobacter baumannii. Clin Microbiol Infect. 2018; 24:908.e1-7. https://doi.org/10.1016/j.cmi.2017.10.033.

23. López-Cortés LE, Cisneros JM, Fernández-Cuenca F, Bou G, Tomás M, Garnacho-Montero J, et al. GEIH/REIPI-Ab2010 group. Monotherapy versus combination therapy for sepsis due to multidrug-resistant Acinetobacter 
baumannii: analysis of a multicentre prospective cohort. J Antimicrob Chemother. 2014;69:3119-26.

24. Özvatan T, Akalın H, Sınırtaş M, Ocakoğlu G, Yılmaz E, Heper Y, et al. Nosocomial Acinetobacter pneumonia: treatment and prognostic factors in 356 cases. Respirology. 2016;21:363-9.

25. Pan S, Huang X, Wang Y, Li L, Zhao C, Yao Z, et al. Efficacy of intravenous plus intrathecal/intracerebral ventricle injection of polymyxin B for postneurosurgical intracranial infections due to MDR/XDR Acinectobacter baumannii: a retrospective cohort study. Antimicrob Resist Infect Control. 2018;7:8. https://doi.org/10.1186/s13756-018-0305-5.

26. Sipahi OR, Mermer S, Demirdal T, Ulu AC, Fillatre P, Ozcem SB, et al. Tigecycline in the treatment of multidrug-resistant Acinetobacter baumannii meningitis: results of the Ege study. Clin Neurol Neurosurg. 2018;172:31-8.

27. Trottier V, Namias N, Pust DG, Nuwayhid Z, Manning R, Marttos AC Jr, et al. Outcomes of Acinetobacter baumannii infection in critically ill surgical patients. Surg Infect. 2007:8:437-43.

28. Fleischman RJ, Adams AL, Hedges JR, Ma OJ, Mullins RJ, Newgard CD. The optimum follow-up period for assessing mortality outcomes in injured older adults. J Am Geriatr Soc. 2010;58:1843-9.

29. Aggarwal R, Dewan A. Comparison of nephrotoxicity of Colistin with Polymyxin B administered in currently recommended doses: a prospective study. Ann Clin Microbiol Antimicrob. 2018;17:15.

30. Nation RL, Li J, Cars O, Couet W, Dudley MN, Kaye KS, et al. Framework for optimisation of the clinical use of colistin and polymyxin B: the Prato polymyxin consensus. Lancet Infect Dis. 2015;15:225-34.

31. Tsuji BT, Pogue JM, Zavascki AP, Paul M, Daikos GL, Forrest A, et al. International Consensus Guidelines for the Optimal Use of the Polymyxins: Endorsed by the American College of Clinical Pharmacy (ACCP), European Society of Clinical Microbiology and Infectious Diseases (ESCMID), Infectious Diseases Society of America (IDSA), International Society for Anti-infective Pharmacology (ISAP), Society of Critical Care Medicine (SCCM), and Society of Infectious Diseases Pharmacists (SIDP). Pharmacotherapy. 2019;39:10-39.

32. Valachis A, Samonis G, Kofteridis DP. The role of aerosolized colistin in the treatment of ventilator-associated pneumonia: a systematic review and metaanalysis. Crit Care Med. 2015:43:527-33.

33. Jang JY, Kwon HY, Choi EH, Lee WY, Shim H, Bae KS. Efficacy and toxicity of high-dose nebulized colistin for critically ill surgical patients with ventilatorassociated pneumonia caused by multidrug-resistant Acinetobacter baumannii. J Crit Care. 2017:40:251-6.

34. Falagas ME, Bliziotis IA, Tam VH. Intraventricular or intrathecal use of polymyxins in patients with gram-negative meningitis: a systematic review of the available evidence. Int J Antimicrob Agents. 2007;29:9-25.

35. Boisson M, Jacobs M, Grégoire N, Gobin P, Marchand S, Couet W, et al. Comparison of intrapulmonary and systemic pharmacokinetics of colistin methanesulfonate (CMS) and colistin after aerosol delivery and intravenous administration of CMS in critically ill patients. Antimicrob Agents Chemother. 2014;58:7331-9.

36. Markantonis SL, Markou N, Fousteri M, Sakellaridis N, Karatzas S, Alamanos I, et al. Penetration of colistin into cerebrospinal fluid. Antimicrob Agents Chemother. 2009:53:4907-10.

37. Liu Q, Li W, Feng Y, Tao C. Efficacy and safety of polymyxins for the treatment of Acinectobacter baumannii infection: a systematic review and meta-analysis. PLoS One. 2014;9:e98091. https://doi.org/10.1371/journal. pone.0098091.

\section{Publisher's Note}

Springer Nature remains neutral with regard to jurisdictional claims in published maps and institutional affiliations.

Ready to submit your research? Choose BMC and benefit from:
- fast, convenient online submission
- thorough peer review by experienced researchers in your field
- rapid publication on acceptance
- support for research data, including large and complex data types
- gold Open Access which fosters wider collaboration and increased citations
- maximum visibility for your research: over 100M website views per year
At BMC, research is always in progress.
Learn more biomedcentral.com/submissions

